ARTICLE

Received 13 Oct 2015 | Accepted 18 Jan 2017 | Published 3 Mar 2017

DOI: $10.1038 /$ ncomms14651

OPEN

\title{
Translational repression by a miniature inverted-repeat transposable element in the $3^{\prime}$ untranslated region
}

Jianqiang Shen ${ }^{1}$, Juhong Liu ${ }^{1}$, Kabin Xie ${ }^{1,2}$, Feng Xing ${ }^{1}$, Fang Xiong ${ }^{1}$, Jinghua Xiao ${ }^{1}$, Xianghua Li ${ }^{1} \&$ Lizhong Xiong $^{1}$

Transposable elements constitute a substantial portion of eukaryotic genomes and contribute to genomic variation, function, and evolution. Miniature inverted-repeat transposable elements (MITEs), as DNA transposons, are widely distributed in plant and animal genomes. Previous studies have suggested that retrotransposons act as translational regulators; however, it remains unknown how host mRNAs are influenced by DNA transposons. Here we report a translational repression mechanism mediated by a stowaway-like MITE (sMITE) embedded in the $3^{\prime}$-untranslated region (3'-UTR) of Ghd2, a member of the CCT (CONSTANS [CO], CO-LIKE and TIMING OF CAB1) gene family in rice. Ghd2 regulates important agronomic traits, including grain number, plant height and heading date. Interestingly, the translational repression of Ghd2 by the sMITE mainly relies on Dicer-like 3a (OsDCL3a). Furthermore, other MITEs in the $3^{\prime}$-UTRs of different rice genes exhibit a similar effect on translational repression, thus suggesting that MITEs may exert a general regulatory function at the translational level.

\footnotetext{
${ }^{1}$ National Key Laboratory of Crop Genetic Improvement and National Center of Plant Gene Research (Wuhan), Huazhong Agricultural University, Wuhan 430070, China. ${ }^{2}$ College of Plant Science and Technology, Huazhong Agricultural University, Wuhan 430070, China. Correspondence and requests for materials should be addressed to L.X. (email: lizhongx@mail.hzau.edu.cn).
} 
$\mathrm{T}$ ransposable elements (TEs), as parasitic DNA, have the ability to spread themselves throughout genomes, causing disruption of gene function and driving genome evolution ${ }^{1,2}$. In eukaryotic genomes, TEs are classified as retrotransposons (Class I) intermediated by RNAs, and DNA transposons (Class II) that move directly in a 'cut and paste' mode ${ }^{3}$. Among these transposons, nonautonomous TEs propagate via their autonomous partners that allow their accumulation in animal and plant genomes ${ }^{4-8}$.

With the advances of genomic and transcriptomic sequencing, TEs, especially retrotransposons ${ }^{9}$, have been identified as gene regulators. In mammalian TE studies, short-interspersed elements (SINEs) or long-interspersed elements (LINEs) were demonstrated to modulate gene transcription through restructuring chromatin epigenetically ${ }^{10,11}$ and function as promoters or enhancers ${ }^{12,13}$. In addition, the retrotransposons, transcribed as part of mRNAs, could influence the alternative splicing of transcripts ${ }^{14,15}$, alternative polyadenylation ${ }^{16,17}$, localization $^{18}$, stability ${ }^{19}$, as well as translation ${ }^{20-23}$. For example, a subset of human transcripts contain inverted repeat Alu elements (IRAlus) in the $3^{\prime}$-UTR, and these elements recruit dsRNA-dependent protein kinase (PKR) to translation initiation factor $2 \alpha$ for subsequent phosphorylation, which ultimately leads to translational repression ${ }^{20,24}$. Meanwhile, the responses of mammalian SINEs to stresses cause translational enhancement ${ }^{25}$. In addition, the function of SINEUPs as long noncoding RNAs in stimulating protein synthesis depends on SINEB2 repeats which are complementary to the $5^{\prime}$-ends of target mRNAs ${ }^{26}$.

As a class of DNA transposons, MITEs constitute $\sim 5.2 \%$ of the rice genome sequence, which are distributed non-randomly throughout the genome and enriched in gene regions in particular ${ }^{27}$. Some MITEs within or near gene-coding regions play regulatory roles in modulating mRNA transcription. Previous studies reported that the DNA transposon mPing tends to insert into the $5^{\prime}$ flanking sequences of genes, causing inducible expression of adjacent genes in response to stresses $^{28}$. In addition, the methylation of MITE-embedded promoters has the potential to silence the transcription of nearby genes. Recently, rice 24-nucleotide (24-nt) small interfering RNA (siRNAs), dependent on OsDCL3a, have been identified as potential silencers for the expression of multiple genes that bear MITEs in their promoter regions ${ }^{29}$. Meanwhile, a previous study reported that a MITE in the promoter would repress the gene expression via RNA-directed DNA methylation and H3K9 dimethylation probably ${ }^{30}$. Other studies also support that MITEs could regulate gene transcription on a genome-wide scale $^{31,32}$. However, it remains unknown whether MITEcontained transcripts could regulate the host gene expression at the translational level in plants.

Here we demonstrate that a stowaway-like MITE represses the protein synthesis of an agronomically important gene $G h d 2$, which controls grain number, plant height and heading date in rice (Oryza sativa). The translational repression by the MITE depends on Dicer-like 3a. In addition, the MITE embedded in $3^{\prime}$-UTR of other genes has the similar function in the translational repression. Our findings reveal an unreported role of DNA transposons in the regulation of gene expression at the translational level.

\section{Results}

Ghd2 functions as a suppressor in flowering time in rice. Previous studies have demonstrated that CCT domain-containing genes regulate diverse biological processes, including developmental controls and environmental responses in plants ${ }^{33-36}$.
Natural variations in CCT domain-containing genes are also associated with agronomic traits and geographic distribution in cereals $^{36-38}$. In an effort to identify agronomically important genes in the CONSTANS-like gene family in rice, we found that a member (LOC_Os02g49880) of this gene family had functions (as the description below) similar to its close homolog Ghd7 (ref. 36), which controls grain number, plant height and heading date. This gene was once simply named $O s K$ in a phylogenetic study ${ }^{39}$. Considering the standard gene nomenclature on the basis of gene-controlled phenotypes in rice ${ }^{40}$, it was renamed Ghd2.

In contrast to other members in the CCT gene family, Ghd2 contains a MITE in its $3^{\prime}$-UTR. Therefore, Ghd2 may be an excellent candidate to investigate the possible roles of MITEs in the gene regulation. This MITE, which is a 217-nt inverted region (IR), was identified as a copy of a stowaway-like MITE designated as stowaway38 $(\mathrm{OsT} 38)^{41}$. By using rapid amplification of cDNA ends (RACE), the transcriptional termination site (TTS) of the Ghd2 mRNA was mapped to 340 nucleotides downstream of the sMITE location (Fig. 1a). To determine whether the sMITE in the Ghd2 3'-UTR has functional significance, we overexpressed the full-length Ghd2, including its $3^{\prime}$-UTR (Ghd2 FL), and the complete coding sequence without the $3^{\prime}$-UTR (Ghd2 FL $\left.\Delta \mathrm{U}\right)$ in the Zhonghua11 rice variety (Fig. 1a). Furthermore, we examined the phenotypes of single-copy transgenic lines. Interestingly, the Ghd2 FL $\Delta \mathrm{U}$ overexpression lines showed approximately 60-day delay in flowering time, whereas the Ghd2 FL overexpression lines exhibited nearly 15-day delay (Fig. $1 \mathrm{~b}$ and Supplementary Table 1). In addition, the Ghd 2 knockout lines with readingframe-shifted mutations generated by the CRISPR/Cas9 technique flowered approximately 7-days earlier than the wildtype (WT) lines (Supplementary Fig. 1b).

A quantitative reverse transcription-PCR (qRT-PCR) analysis demonstrated that the Ghd2 gene was highly expressed in leaves at the reproductive stage (Supplementary Fig. 2) and the expression peaked at 20:00 during a diurnal cycle (Supplementary Fig. 3a and b). Furthermore, the expression level of the Early heading date 1 (Ehd1) gene $\mathrm{e}^{42}$, but not Heading date $1(\mathrm{Hd} 1)^{43}$, was dramatically reduced in the Ghd2 FL $\Delta \mathrm{U}$ overexpression lines, which suggested that Ghd2 may function as a suppressor of Ehd1, thus delaying flowering time in rice (Supplementary Fig. 3e and $\mathrm{f}$ ). In addition to the delayed flowering time, Ghd2 overexpression caused a significant increase in panicle size, the number of spikelets and grain yield (Fig. 1b), which is similar to the phenotypes of the Ghd7 overexpression transgenic rice.

The sMITE functions as a translational repressor. The phenotypic variance between $G h d 2 \mathrm{FL}$ and $G h d 2 \mathrm{FL} \Delta \mathrm{U}$ prompted us to propose that the Ghd2 $3^{\prime}$-UTR may function as a repressor. To test this hypothesis, we quantified the Ghd2 transcript and protein levels in the Ghd2 FL and Ghd2 FL $\Delta \mathrm{U}$ overexpression lines. Interestingly, equivalent levels of Ghd2 mRNA were observed in the Ghd2 FL and Ghd2 FL $\Delta \mathrm{U}$ overexpression lines (Fig. 1c), but the Ghd2 protein level was higher in the Ghd2 FL $\Delta \mathrm{U}$ overexpression line (3-fold increase compared to the WT) than that in the Ghd2 FL overexpression line (1.6-fold increase compared to the WT; Fig. 1d). These results imply that the $3^{\prime}$-UTR may regulate $G h d 2$ at the translational level.

To investigate whether the sMITE regulates Ghd2 translation directly, we transiently expressed the Ghd2 $3^{\prime}$-UTR in rice protoplasts using a dual luciferase reporter assay. Constructs of a firefly luciferase gene bearing the Ghd2 3 '-UTR deletion forms or the cauliflower mosaic virus $35 \mathrm{~S}$ terminator (35S Ter) were used as reporters. A Renilla luciferase gene with $35 \mathrm{~S}$ Ter in 

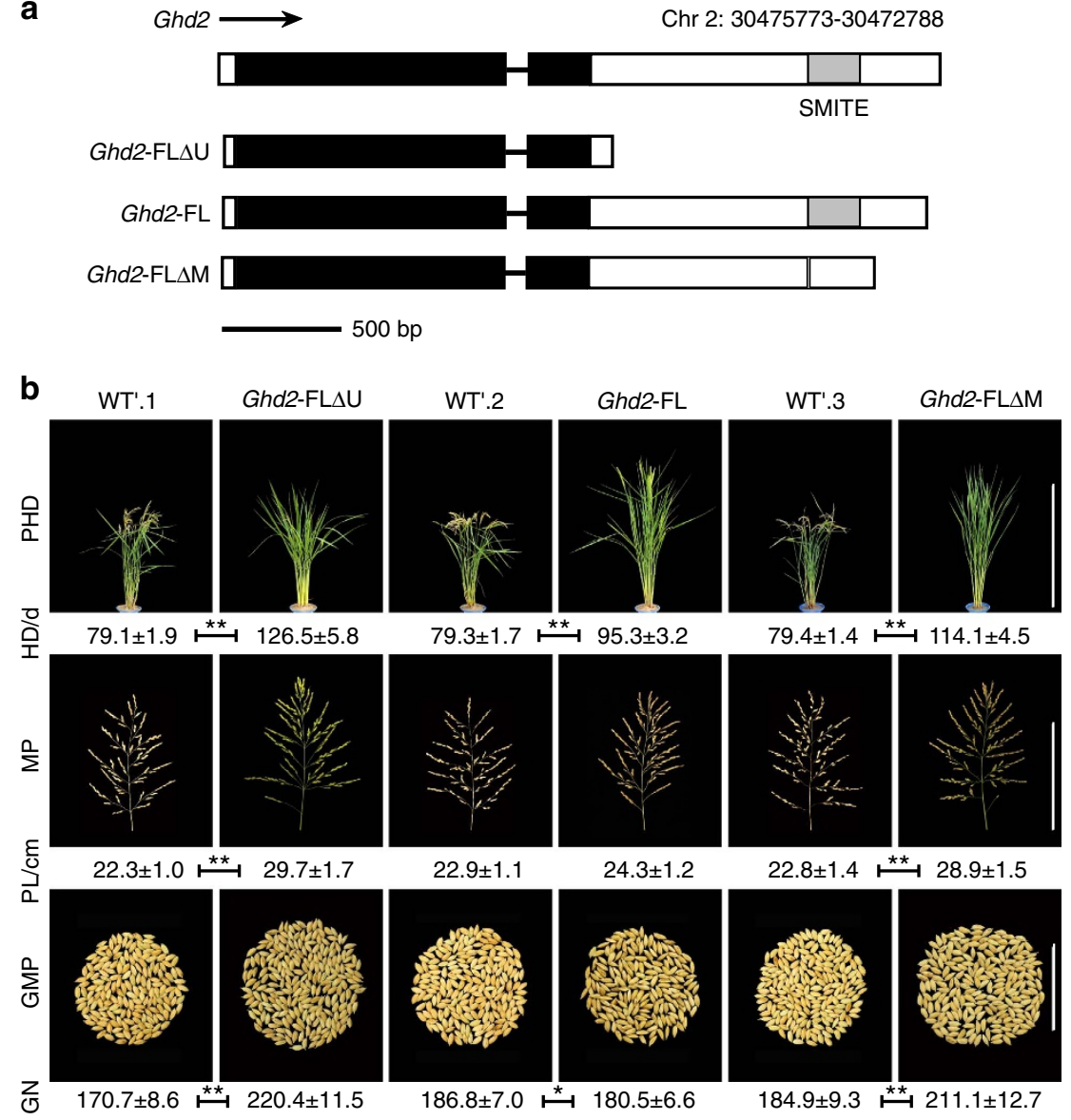

C

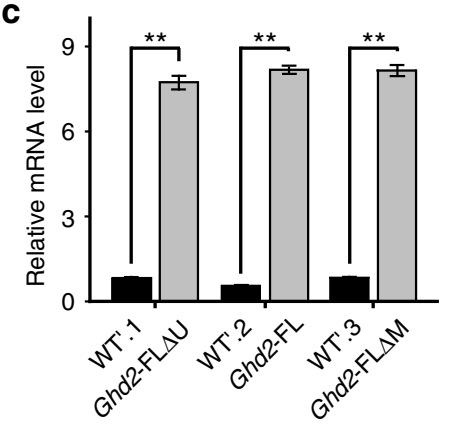

d

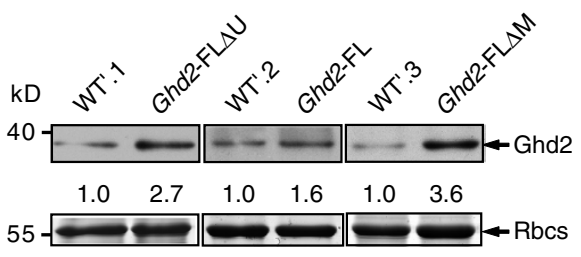

Figure 1 | The Ghd2 3'-UTR acts as a negative regulator of flowering and yield. (a) Ghd2 genomic organization. Exons are in black; $3^{\prime}-$ and 5'-UTRs are in white; the transposable element (sMITE) is in grey; and the intron is indicated as a line. (b) Phenotypes of Ghd2-FL $\Delta \mathrm{U}$ overexpression and wild-type (WT'1) lines, Ghd2-FL overexpression and wild-type (WT'2) lines, and Ghd2-FL $\Delta M$ overexpression and wild-type (WT'3) lines. GN, grain number of main panicle; GMP, grains of main panicle; HD, heading date (days); MP, main panicle; MPL, main panicle length ( $\mathrm{cm}$ ); PHD, phenotype of heading date at the maturation stage of WT. Scale bar: $1 \mathrm{~m}$ (PHD), $20 \mathrm{~cm}$ (MP), $10 \mathrm{~cm}$ (GMP). WT' indicates segregated non-transgenic lines. The data represent the means \pm s.d., $n=20$. ${ }^{\star} P<0.05,{ }^{*} P<0.01$ (Student's t-test). (c) Quantitative expression of Ghd2 mRNA in Ghd2-FL $\Delta \mathrm{U}$ and WT'1; Ghd2-FL and WT'2; and Ghd2-FL $\Delta M$ and WT'3 lines. The data represent the means \pm s.d., $n=3$. ${ }^{\star \star} P<0.01$ (Student's $t$-test). (d) Ghd2 protein levels in Ghd2-FL $\Delta U$ and WT'1; Ghd2-FL and WT'2; and Ghd2-FL $\Delta \mathrm{M}$ and WT'3 lines were measured by western blotting with an anti-Ghd2 antibody. Rubisco (Rbcs) was loaded as a control. The numbers between two blots indicate relative abundance of Ghd2 normalized by Rbcs for single samples.

its $3^{\prime}$-UTR was co-transfected to normalize the transfection efficiency (Fig. 2a). The relative luminescence normalized to the mRNA level of the luciferase gene (relative luminescence/ mRNA level) was used to evaluate the relative translational efficiency. The results showed that the complete Ghd2 3 '-UTR and the shortened $3^{\prime}$-UTRs containing the sMITE significantly repressed luciferase expression, but the partial $3^{\prime}$ UTRs lacking the sMITE had no repression effect (Fig. 2a). Furthermore, the constructs with sMITE substituted by an unrelated sequence
(GUS.1) or another retrotransposon (GUS.2) were assayed. Compared to the construct with the complete $3^{\prime}$ UTR, the constructs with GUS.1 or GUS.2 exhibited an approximate 3-fold increase in the relative luminescence/mRNA level (Fig. 2a). These results indicate that the sMITE is a functional TE required for the 3'-UTR-mediated translational repression of Ghd2. To further confirm the results of the transient expression, we overexpressed the full-length Ghd2 gene without the sMITE (Ghd2 FLAM) in rice. As expected, the single-copy transgenic lines of Ghd2 
a
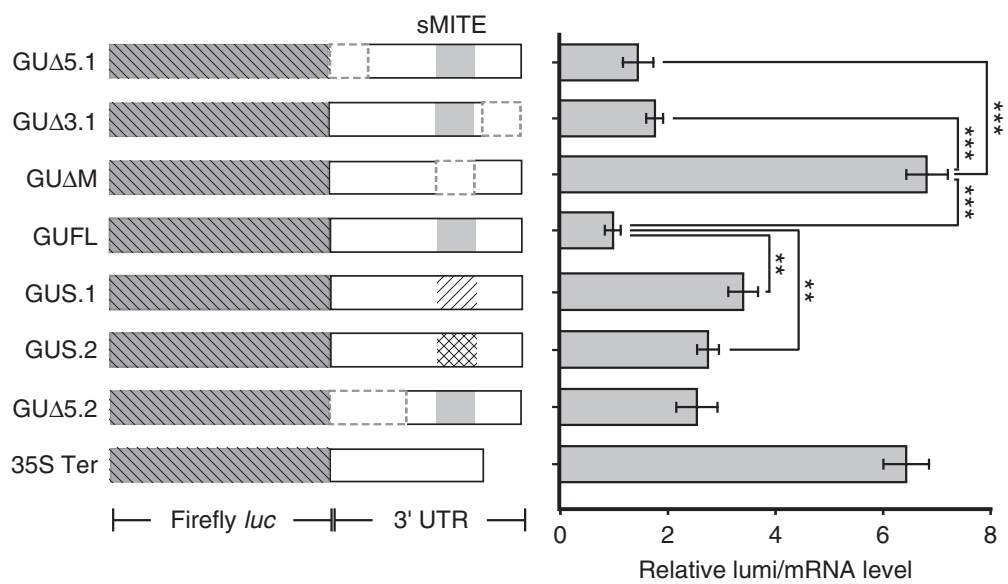

b
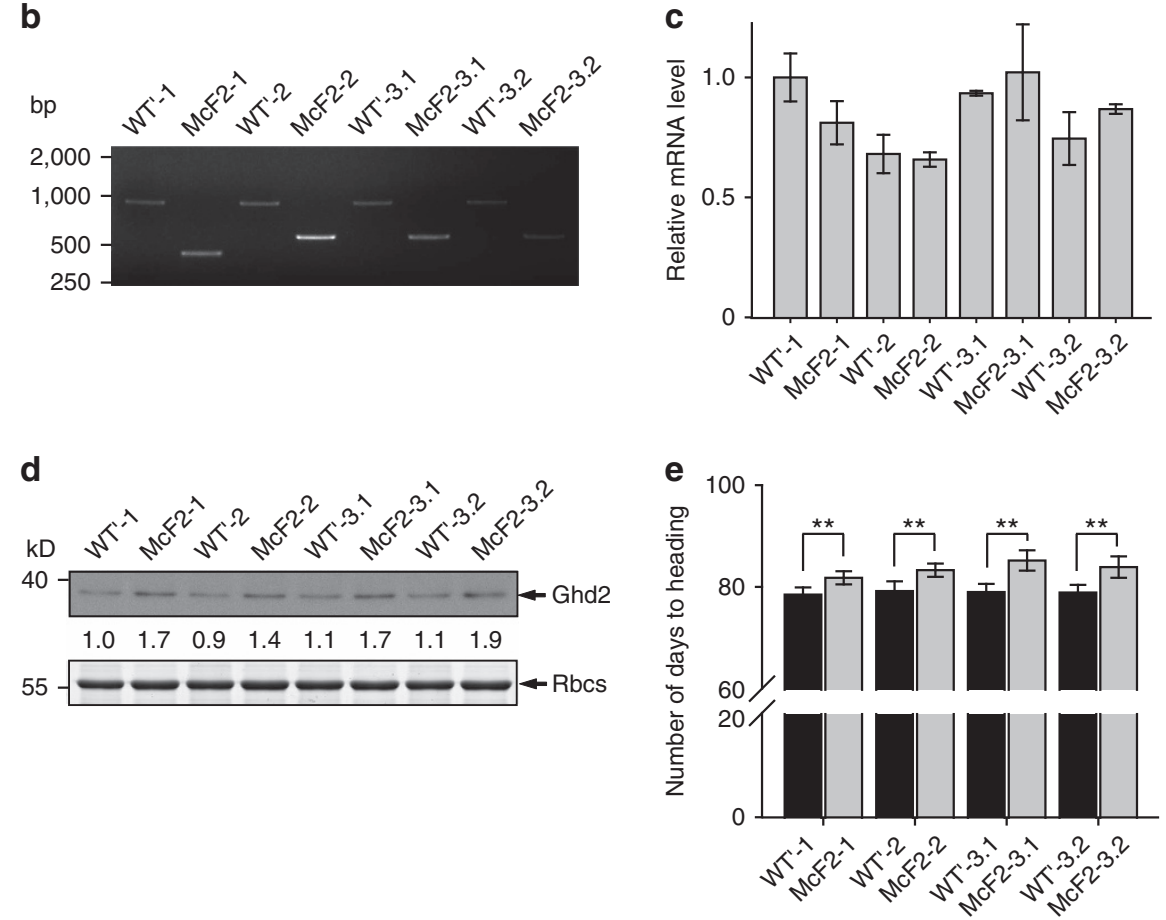

Figure 2 | The Ghd2 $\mathbf{3}^{\prime}$-UTR regulates the Ghd2 protein level via a sMITE. (a) Left panel, firefly luciferase reporter constructs fusing the full-length Ghd2 3'-UTR (GUFL), deletion variants (GU $\Delta 5.1, \mathrm{GU} \Delta 3.1, \mathrm{GU} \Delta \mathrm{M}$ and GU $\Delta 5.2$ ), substitute variants (GUS.1 and GUS.2), and the cauliflower mosaic virus $35 \mathrm{~S}$ terminator (35S Ter, control). The luciferase genes are in dark grey with diagonals; the $3^{\prime}$-UTRs are in white, the deleted regions are shown with dotted lines, the variants are in light grey with diagonals; and the grey rectangle in the $3^{\prime}$-UTR indicates the sMITE. Right panel, relative luminescence units versus the transcript levels quantified by qPCR (GUFL was normalized to 1). The data in the right panel represent the means \pm s.d., $n=3$. ${ }^{\star \star} P<0.01$ (Student's t-test). The data indicate the relative luminescence/mRNA level calculated from three independent experiments. (b) Genotypes of the multi-sgRNA-mediated transposable element excision lines. Three independent sMITE-excision lines containing 519,397 and 397 bp deletion, respectively in the Ghd2 3' UTR were backcrossed with wild type to generate the segregated excision (McF2-1, McF2-2, and McF2-3) and non-excision (WT') lines. McF2-3.1 and McF2-3.2 are two backcrossed excision lines from the same transgenic events. (c) Quantitative expression of Ghd2 mRNA in the sMITE excision lines and negative controls. The data represent the means \pm s.d., $n=3$. (d) The Ghd 2 protein level in the sMITE excision lines and negative controls was measured by western blotting with the anti-Ghd2 antibody. Rubisco (Rbcs) was loaded as a control. The numbers between two blots indicate relative abundance normalized by Rbcs for single samples. (e) The heading date of MITE excision (McF2) and the segregated wild-type (WT') lines. The data represent the means \pm s.d., $n=10$. ${ }^{\star \star} P<0.01$ (Student's $t$-test).

FL $\Delta \mathrm{M}$ and Ghd2 FL $\Delta \mathrm{U}$ exhibited similar phenotypes (Fig. 1b), Ghd2 mRNA levels (Fig. 1c), and Ghd2 protein abundance (Fig. 1d).

To further clarify the regulatory role of sMITE in Ghd2 translation, we generated transgenic rice lines, in which the sMITE genomic sequence was precisely excised from the Ghd2 3'-UTR using CRISPR/Cas9 editing (Fig. $2 \mathrm{~b}$ and
Supplementary Fig. 4a). To segregate the foreign DNA from the sMITE excision plants, three $\mathrm{T}_{2}$ excision lines with two different sizes of excision fragment (Supplementary Fig. 4b) were backcrossed with the wild type. The segregated sMITE excision plants (named McF2-1, McF2-2 and McF2-3) and the wild type (WT') from $\mathrm{BC}_{1} \mathrm{~F}_{2}$ generation were identified for Ghd2 expression level analysis. The Ghd2 mRNA levels in the sMITE- 
excised lines showed no significant change compared to that in the non-excision lines (Fig. 2c and Supplementary Fig. 4c), while the Ghd2 protein levels in the sMITE-excised lines were obviously higher (Fig. 2d and Supplementary Fig. 4d). Meanwhile, the flowering time of the excision lines showed approximately 5-day delay compared to that of the wild type (Fig. 2e). These results further confirmed that Ghd2 translation is repressed by the sMITE in its $3^{\prime}$-UTR.
Translational repression by the sMITE depends on OsDCL3a. It has been reported that transposable element-derived siRNAs mediate chromatin modification to regulate the abundance of FLC mRNA ${ }^{44,45}$ and alter ribosome sensitivity to repress translation ${ }^{46}$. We identified two siRNA clusters, siR381 and siR382, derived from the sMITE using the Cereal Small RNA database ${ }^{47}$. To investigate whether these two siRNAs are involved in the Ghd2 translational repression, we overexpressed

a

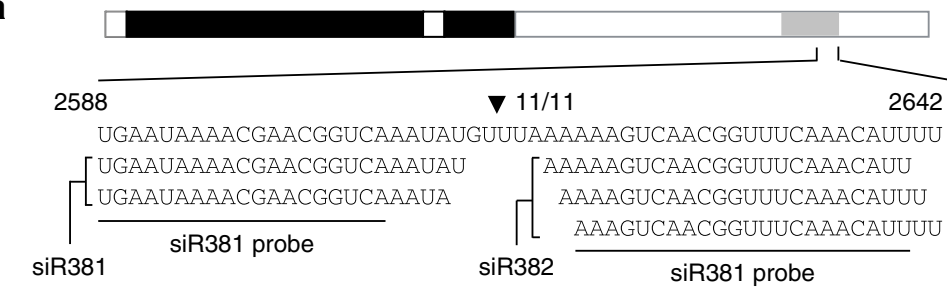

bM

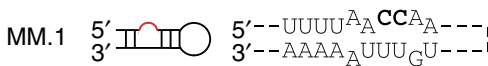

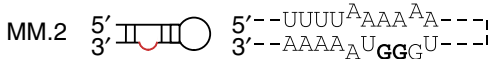

$5^{\prime}$
$5^{\prime}$

d

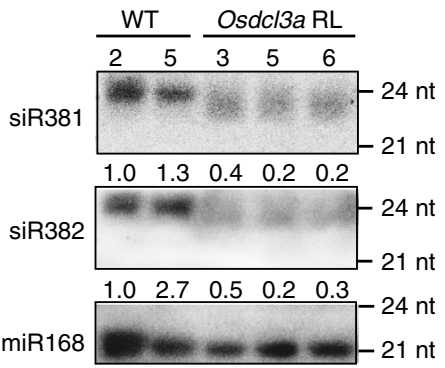

f
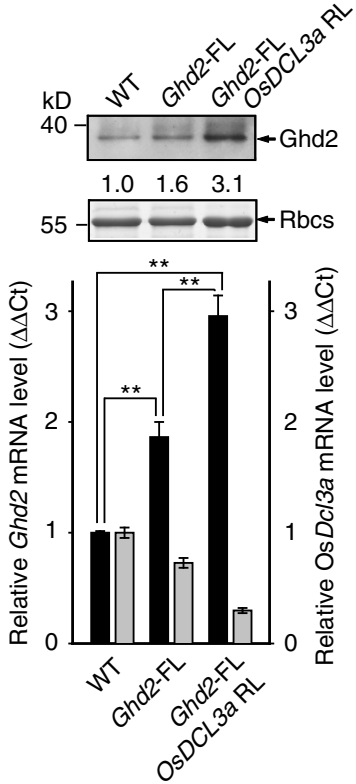

c

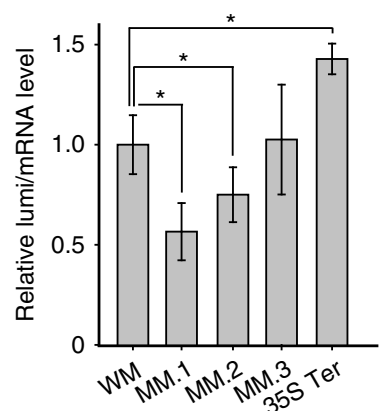

e
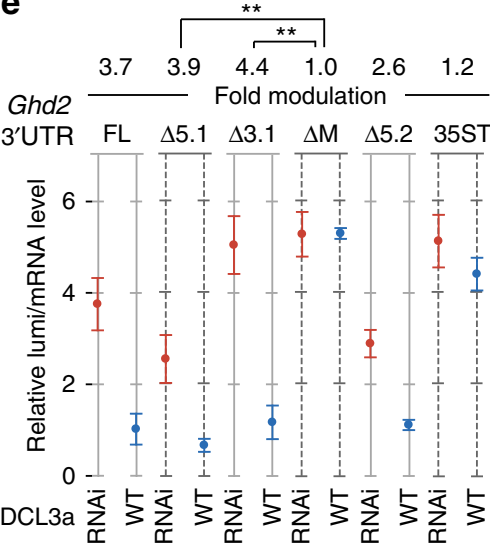

g WT Ghd2-FL $\quad$ Ghd2-FL

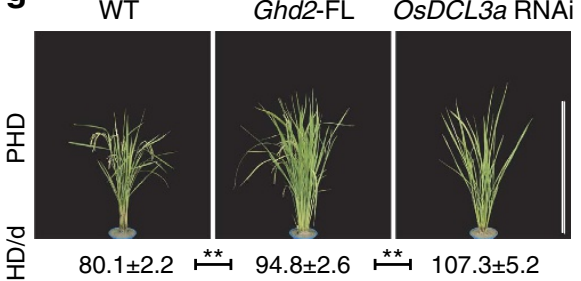


them in rice. Surprisingly, neither the Ghd 2 mRNA nor the Ghd2 protein level showed significant differences between the siRNA overexpression lines and the WT lines (Supplementary Fig. 5). These results suggest that the translational repression may not be related to the abundance of the siRNAs derived from the sMITE.

We further examined whether the translational repression of Ghd 2 was associated with the siRNA biogenesis. A modified RNA ligase-mediated rapid amplification of $5^{\prime} \mathrm{cDNA}$ ends (RLM-RACE) was employed to examine the target site in Ghd2 3'-UTR transcripts in vivo. The result revealed the presence of a cleavage site, which is located between the two siRNA sequences (Fig. 3a). To assess the importance of this site for translation, the sMITE and its mutated forms of the site (Fig. 3b) were fused to the $3^{\prime}$-UTR of firefly luciferase gene for expression assay. Compared to the control (35S Ter), the luciferase translation was repressed in the protoplasts transfected with the wild-type sMITE construct (WM; Fig. 3c). The translational repression in the protoplasts transfected with the mutant constructs (MM.1 and MM.2) was enhanced compared to that with the WM (Fig. 3c). This result may be due to the mutations that are possibly more effectively recognized and cleaved by endoribonucleases, such as dicer-like proteins (DCL) ${ }^{48}$.

The increased suppression of the mutated luciferase reporters prompted us to investigate whether endoribonucleases, which process the siRNAs, are responsible for the translational repression. Small RNA northern blot analyses were preformed in RNA interference (RNAi) or mutant lines of Dicer-like endoribonuclease genes including OsDCL1, OsDCL3a, OsDCL3b and OsDCL4. Only in the OsDCL3a RNAi lines, the abundance of the sMITE-derived 24 nt-siRNAs was attenuated (Fig. 3d and Supplementary Fig. 6). The results revealed that OsDCL3a may act as a regulator for the sMITE-derived siRNAs. Heterochromatic siRNAs are derived from double-stranded RNA (dsRNA) generated by RDR2, processed by DCL3 and incorporated into AGO4 in plants ${ }^{49-52}$. Therefore, we speculated that some of the proteins involved in siRNA biogenesis and binding, such as OsRDR2, OsDCL3a, OsAGO4a and OsAGO4b might be involved in sMITE-mediated translational regulation ${ }^{31}$. This hypothesis was tested by examining the Ghd 2 transcript and protein abundance in the mutant or RNAi lines of these genes. Interestingly, compared with the WT line, the OsDCL1, $O s D C L 3 a$ and $O s D C L 3 b$ RNAi lines exhibited an increased accumulation of the Ghd2 protein, but not the Ghd2 transcripts (Supplementary Fig. 7a,b, and c); however, Ghd2 protein was decreased in the $d c l 4$ mutant (Supplementary Fig. 7d). The knockdown of OsRDR2 and OsAGO4ab had no effect on Ghd2 protein accumulation (Supplementary Fig. 8). All these results indicate that OsDCL3a may be involved in the sMITE-mediated translational repression.
To further confirm this hypothesis, we assayed the relative luminescence/mRNA level of the reporter gene containing the sMITE in the $3^{\prime}$-UTR in the Osdcl3a-RNAi line and WT protoplasts. The relative luminescence/mRNA level of the reporter gene was significantly higher in the Osdcl3a-RNAi lines than in the WT (Fig. 3e). The results indicate that translational repression was weakened in the OsDCL3a RNAi protoplast. Furthermore, the knockdown of OsDCL3a in the Ghd2 FL transgenic plants resulted in phenotypes similar to that of Ghd2 FL $\Delta \mathrm{M}$ (Fig. $3 \mathrm{~g}$ ). The Ghd2 protein level was higher in the OsDCL3a-RNAi Ghd2 FL plants than in the Ghd2 FL plants (Fig. 3f). Interestingly, the Ghd2 transcript level was increased in the OsDCL3a RNAi line, suggesting that the Ghd 2 mRNA may be more stable because of OsDCL3a deficiency (Fig. 3f). These results together suggest that OsDCL3a is responsible for Ghd2 translational repression mediated by the sMITE.

MITEs in other $3^{\prime}$-UTRs also repress translation. MITEs associated with genes are broadly distributed in the rice genome ${ }^{41}$. For example, the sMITE in this study (OsT38) has $\sim 4,518$ copies (Supplementary Fig. 9). We further investigated whether other MITE-embedded $3^{\prime}$-UTRs have similar roles in the repression of protein synthesis. To find more genes with an insertion of MITE in their $3^{\prime}$-UTR, MITEs identified in the rice genome ${ }^{53}$ were scanned for protein-encoding genes containing a MITE in their $3^{\prime}$-UTRs. A total of 1,182 genes were identified with $3^{\prime}$-UTRs embedded with 1,694 MITEs potentially generating 23- to 24-nt siRNAs (Fig. 4a). Three $3^{\prime}$-UTRs with MITEs other than OsT38 were randomly chosen, fused to the $3^{\prime}$-UTR of the luciferase reporter, and transfected into rice protoplasts. All three $3^{\prime}$-UTRs prominently repressed translation when compared with a control reporter carrying the $35 \mathrm{~S}$ terminator (Fig. $4 \mathrm{~b}$ ). These results imply that a large number of plant genes may be regulated by MITEs at the translational level.

\section{Discussion}

In eukaryotic genomes, retrotransposons have been found to have multiple functions in regulating genes expression and are known to participate in various metabolic processes 9 . In animal and plant genomes, MITEs (a type of nonautonomous DNA transposons) constitute $0.32-15.8 \%$ of the genomic sequences ${ }^{54}$. These MITEs are found in untranslated regions of mRNA including $5^{\prime}$-UTRs, introns and $3^{\prime}$-UTRs ${ }^{27}$. Extensive evidence supported that the MITEs present upstream of adjacent genes can alter their transcription as promoters ${ }^{28}$ or through epigenetic modifications ${ }^{29}$. However, little is known about the functions of MITEs in the translational modulation despite the predominant regulatory roles of MITEs at the transcriptional level. In this study, we found that a stowaway-like MITE

\footnotetext{
Figure 3 | SMITE-mediated Ghd2 translational repression depends on OsDCL3a. (a) The cleavage site in the sMITE (indicated by a black triangle) was mapped by RLM-5' RACE. The sequence indicates the 2588 to $2643 \mathrm{nt}$ of the Ghd2. The small RNA sequences below were aligned to the sMITE in the Ghd2 3'-UTR. Probes used in the small RNA northern blotting are underlined. (b) Predicted secondary structure of the wild-type (MW) and mutant (MM.1-MM.3) sites. The right panel shows the site mutants (bold). (c) Relative luminescence units versus the transcript levels normalized to MW (set as 1). The data represent the means \pm s.d., $n=3 .{ }^{*} P<0.05$ (Student's $t$-test). The data indicate the relative abundance calculated from three independent experiments. (d) Decreased levels of siRNAs from the sMITE in the Osdcl3a RNAi lines (RL). The probes for the siRNAs are as shown in a. MiR168 was used as a loading control. The numbers below the blots indicate the relative abundance. (e) Dual luciferase reporter assay of the Ghd2 $3^{\prime}$ UTR and its deletion variants in the Osdcl3a RNAi lines and the WT. Relative luminescence units versus their relative mRNA level are shown (normalized to GUFL). Fold modulation means the relative luminescence/mRNA level of OsDCL3a RNAi line versus the WT. The data represent the means \pm s.d., $n=3$. ${ }^{\star \star} * P<0.01$ (Student's $t$-test). (f) The Ghd2 mRNA and protein levels were quantified in the WT, Ghd2 FL and OsDCL3a-RNAi Ghd2 FL lines. The Ghd2 protein was measured by western blotting using the anti-Ghd2 antibody. Rubisco (Rbcs) was loaded as a control. The numbers between two blots indicate relative abundance for single samples. The expression of Ghd2 mRNA was quantified by quantitative RT-PCR. The data represent the means \pm s.d., $n=3 .{ }^{\star \star} P<0.01$ (Student's t-test). (g) Phenotypes of the WT, Ghd2 FL and OsDCL3a-RNAi Ghd2 FL lines. HD, heading date (days); PHD, phenotype of the heading date at the maturation stage of the WT (scale bar, $50 \mathrm{~cm}$ ). The data represent the means \pm s.d., $n=10$. ${ }^{\star \star} P<0.01$ (Student's $t$-test).
} 

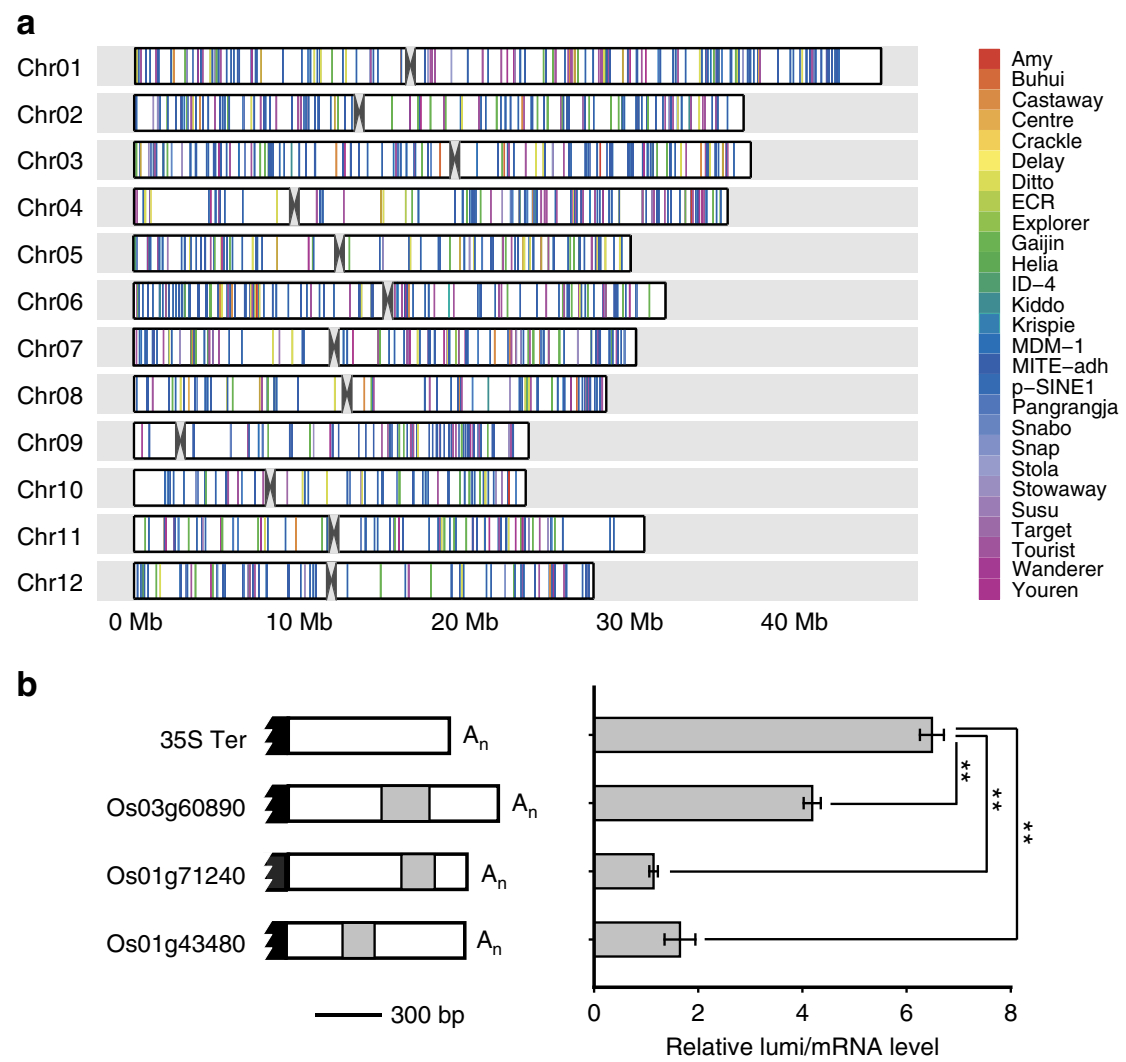

Figure 4 | Other MITEs in $\mathbf{3}^{\prime}$-UTRs repress translation. (a) Genomic distribution of $3^{\prime}$ UTR-localized MITEs in the rice genome. A karyogram composed of colored bars is presented; each colored bar represents a MITE in the 3' UTR of the target gene. Black angles represent centromeres. (b) Genomic organization of three randomly chosen MITEs in $3^{\prime}$-UTRs (left) and their effects on translational repression in rice protoplasts (right). The data represent the means \pm s.d., $n=3$. ${ }^{\star} P<0.01$ (Student's $t$-test). The data indicate relative luminescence/mRNA level based on three independent experiments.

functions in the translational repression of the Ghd2 gene, which controls grain number, plant height, and heading date. Furthermore, this translational repression is dependent on OsDCL3a. Based on our results, we propose a simple model for the role of this MITE in the translational repression (Supplementary Fig. 10). Besides Ghd2, a genome-wide search identified that 1,182 genes harbour MITEs in their $3^{\prime}$-UTRs, and these genes also appear to be modulated also through MITE-mediated translational repression.

In this study, we found that the MITE-mediated translational repression is dependent on OsDCL3a. It remains unknown which phase of mRNA translation is blocked and how the translation is repressed. Most probably, OsDCL3a may direct the translational repression by processing the MITE nascent transcripts, thus generating a shortened mRNA. The shortened mRNA or the loss of a polyadenylation tail may be a trigger to the translational repression. Several lines of evidence support this hypothesis. First, the cleavage in the MITE suggests that the Ghd2 mRNA is truncated by OsDCL3a, which was confirmed by the result of dual-luciferase reporter assay with the constructs mutated at the cleavage site (Fig. 3a,b). Second, the Ghd2 mRNA levels in the OsDCL3a-RNAi Ghd2 FL lines were higher than that in the Ghd2 FL overexpression lines (Fig. 3f). This indicates that the mRNA truncation can be attenuated due to the suppression of OsDCL3a. Previous study reported that the 24-nt siRNAs derived from transposon transcripts processed by OsDCL3a mediate DNA methylation and result in histone modification (H3K9 dimethylation) in rice ${ }^{29}$. To check whether the DNA methylation and/or H3K9 dimethylation are affected by the MITE at the Ghd2 locus, bisulfite sequencing and chromatin immunoprecipitation combined with quantitative PCR (ChIP-qPCR) were employed in the MITE excision lines and the wild types. According to the bisulfite sequencing data and the ChIP-qPCR results, the DNA methylation and H3K9 dimethylation level at the Ghd2 locus showed no significant difference between the MITE excision lines and wild types with the MITE (Supplementary Fig. 11). Meanwhile, our data suggested that the Ghd2 transcript level is not affected by the presence or absence of MITE (Figs 1c and 2c), indicating that the MITE excision may not affect the transcription level of this gene.

It should be noted that 21-22-nt small RNAs were observed in the siRNA-overexpression lines and in the DCL3a RNAi lines (Fig. 3d and Supplementary Fig. 5a and b). It is possible that other Dicer-like proteins are involved to generate distinct sizes of small RNAs, likely through their coordinated actions with OsDCL3a (ref. 31). Previous data showed both 24-nt miRNAs and 21-nt miRNAs, derived from the same miRNA precursor, would direct DNA methylation and RNA cleavage, respectively ${ }^{31}$. These $21-$ 22-nt small RNAs may be possible clues to further unveiling the MITE-mediated translational repression.

The sMITE-mediated Ghd2 translational repression provides a novel mechanism to prevent the excessive accumulation of the Ghd2 protein in addition to the transcriptional regulation. As a gene controlling multiple agronomic traits, Ghd2 is conserved in rice subspecies including indica and japonica. Additionally, we analysed the genomic sequence of Ghd2 in wild rice, and found that the MITE in the Ghd2 $3^{\prime}$ UTR is conserved in the wild relatives of the Asian cultivated rice, O. rufipogon, but not in the wild rice O. meridionalis (Supplementary Fig. 12). 
Taken together, our findings reveal that MITE-mediated translational repression is an unreported but important mechanism for fine tuning gene expression at the translational level (Supplementary Fig. 10). Gene-specific regulation by MITEs, especially for agronomically important genes such as Ghd2, may represent an outcome of evolutionary pressure on the genomic organization of transposable elements. The MITE embedded in the Ghd2 3'-UTR, which regulates the protein level of Ghd2, may represent a promising engineering tool for controlling the protein levels of other agronomically important genes.

\section{Methods}

Plant materials. Oryza sativa L. ssp. japonica var. Zhonghual1 was used to generate Ghd2 overexpression, Ghd2 CRISPR, and MITE excision transgenic lines using Agrobacterium-mediated transformation ${ }^{55}$. Rice calli were induced from matured embryo. After subculture, the calli were co-cultured with Agrobacterium strain EHA105 containing the constructs described below. The transformed calli were selected on the basis of hygromycin B (Roche) or G-418 (Promega) resistance to generate transgenic plants. In all of the transgenic lines, the copy numbers of marker genes for transformation were determined by Southern blot. Two to three independent single-copy lines and the segregated non-transgenic lines were used for phenotypic analysis in the $\mathrm{T}_{2}$ or $\mathrm{T}_{3}$ generation (Supplementary Table 1). To generate MITE excision lines with background close to the wild type, three independent $\mathrm{T}_{2}$ transgenic MITE excision plants were backcrossed with the wild type rice Zhonghua11. The backcrossed MITE excision plants (McF2) and non-excision plants $\left(\mathrm{WT}^{\prime}\right)$ segregated from $\mathrm{BC}_{1} \mathrm{~F}_{2}$ generation were identified for further experiments.

The OsDCL1, OsDCL3a, OsDCL3b, OsRDR2 and OsAGO4ab RNAi lines and the osdcl4 mutant were obtained from the authors of previous reports ${ }^{31,56,57}$. The wild rice materials were from Prof. Qifa Zhang's lab and the cultivated rice DNA was provided by the authors of a previous report ${ }^{58}$. The OsDCL3a RNAi construct $^{31}$ was introduced into the Ghd2 FL overexpression lines to obtain the OsDCL3a-RNAi Ghd2 FL overexpression lines.

Phenotypic analysis. All of the transgenic lines were grown during natural rice growing seasons in the experimental fields of Huazhong Agricultural University $\left(114.36^{\circ} \mathrm{E}, 30.48^{\circ} \mathrm{N}\right)$, Wuhan, China. For the diurnal expression pattern analysis, 15-day-old seedlings of the overexpression lines and the WT lines were cultivated in a phytotron (PGV36, Conviron) at $60 \%$ humidity under long-day conditions (cycles of $14 \mathrm{~h}$ of light at $30^{\circ} \mathrm{C}$ and $10 \mathrm{~h}$ of dark at $26^{\circ} \mathrm{C}$ ) or under short-day conditions (cycles of $10 \mathrm{~h}$ of light at $30^{\circ} \mathrm{C}$ and $14 \mathrm{~h}$ of dark at $26^{\circ} \mathrm{C}$ ).

The flowering time (heading date) was the days from the germination to the spikelet head-out. The plant height was measured by the height from the ground surface to the top of the plant at the mature stage. The main panicle length and main panicle spikelet number was determined after collection. All of the phenotypic traits were measured for at least ten plants of each genotype with two replicates. The Student's $t$-test was used for statistical analysis.

Protoplast isolation. For transient expression in rice protoplasts, 3-day-old seedlings of Zhonghual1 were planted on 1/2 MS medium and were cultivated in an illumination room at $60 \%$ humidity, with $14 \mathrm{~h}$ of light at $28^{\circ} \mathrm{C}$ and $10 \mathrm{~h}$ of dark at $25^{\circ} \mathrm{C}$. Protoplast isolation was performed with 12 to 15 -day-old seedlings $\mathrm{s}^{59}$. The leaf sheaths of seedlings were digested with $1.5 \%$ Cellulase R-10 (Yacult Pharmaceutical) and 0.75\% Macerozume R-10 (Yacult Pharmaceutical) for $5 \mathrm{~h}$. After filtering through $40 \mu \mathrm{m}$ nylon mesh, the protoplasts were collected and incubated in the W5 solution $\left(154 \mathrm{mM} \mathrm{NaCl}, 5 \mathrm{mM} \mathrm{KCl}, 125 \mathrm{mM} \mathrm{CaCl}_{2}\right.$, $22 \mathrm{mM}$ MES, pH 5.7) at room temperature for at least $1 \mathrm{~h}$. After incubation, the protoplasts were resuspended in the MMG solution ( $0.6 \mathrm{M}$ mannitol, $15 \mathrm{mM}$ $\mathrm{MgCl}_{2}, 4 \mathrm{mM}$ MES, $\mathrm{pH} 5.7$ ), and the concentration of protoplasts was adjusted to the $1 \times 10^{7} \mathrm{ml}^{-1}$ for PEG-CaCl 2 mediated transfection.

Genotypic analysis. The genotypes of Ghd 2 and siRNA overexpression lines were identified by genomic PCR using the Hn forward and reverse primers (Supplementary Table 2). The genotypes of OsDCL1, OsDCL3a, OsDCL3b, OsRDR2, OsAGO4ab RNAi lines and osdcl mutants were identified as per the methods utilized in previous reports ${ }^{31,56,57}$. The genotypes of MITE excision lines, wild rice, and rice cultivars were identified by genomic PCR using the MEC forward and reverse primers and the amplified genomic DNA was confirmed by sequencing (Fig. $2 \mathrm{~b}$ and Supplementary Fig. 4b). The genotypes of Ghd2 CRISPR lines were identified by sequencing using the Ghd2 crispr forward and reverse primer (Supplementary Fig. 1a). The foreigner DNA in the MITE excision lines was identified by PCR using the Cas9 forward and reverse primers (Supplementary Table 2).

Plasmid construction. A full-length DNA fragment of $G h d 2$ was amplified from Zhonghual1 genomic DNA with the Ghd $2 \mathrm{fl}$ forward and reverse primers. The full-length Ghd2 without the $3^{\prime}$-UTR was amplified from the Ghd2 full-length fragment with the Ghd2 cds forward and reverse primers. The full-length Ghd2 without the MITE was generated via fusion PCR using the Ghd2 $\mathrm{fl}$ primers and Ghd2 fldM inside primers. All of the amplified fragments were cloned into pCAMBIA1301H, in which the Ghd2 genes were driven by the OsLEA3 promoter ${ }^{60}$. To generate the Ghd2 CRISPR lines, fragments containing single guide RNA (sgRNA) were amplified with the Ghd2 crispr primers and were cloned into pH-Ubi-cas9-7 (ref. 61). The sgRNA was designed to target the Ghd2 coding region (Supplementary Fig. 1a).

For the multi-sgRNA-directed transposable element excision, four sgRNAs flanking the sMITE were designed according to the CRISPR-PLANT database (www.genome.arizona.edu/crispr/) ${ }^{62}$. The locations and sequences of guide RNAs used for the CRIPSR construct were shown in Supplementary Fig. $4 \mathrm{a}$ and $\mathrm{b}$. The transfer RNA and guide RNA scaffold were amplified from the pGTR vector with the Ghd2_TED primers (Supplementary Table 2), assembled using golden gate assembly strategy, and ligated into to the pRGEB32 vector ${ }^{63}$ for the Agrobacterium-mediated rice transformation.

For siRNAs overexpression, the OsmiR440 precursor was amplified from the rice genome with the OsmiR440 forward and reverse primers. The mature OsmiR440 sequence was replaced by siR381 and siR382 to construct artificial siRNA precursors with the siR381 and siR382 primers (Supplementary Table 2$)^{64,65}$. The pCAMBIA1301U was employed to carry the artificial siRNA precusors, driven by the maize ubiquitin promoter ${ }^{66}$.

The reporter in p2CGW7 was replaced with the firefly or Renilla luciferase to generate p2FLGW7 or p2RLGW7 for the $3^{\prime}$-UTR assay with the Fluc $\mathrm{fl}$ and Rluc fl primers. The Ghd2 $3^{\prime}$-UTR was obtained by PCR with the GU fl forward and reverse primers. The Ghd2 $3^{\prime}$ UTR deletion mutants were generated from the full-length Ghd2 $3^{\prime}$-UTR with the primers listed in Supplementary Table 2. The Ghd2 $3^{\prime}$-UTR $\Delta \mathrm{M}$ was generated with a strategy similar to that used for the Ghd2 FL $\Delta \mathrm{M}$. The $35 \mathrm{~S}$ terminator, a negative control, was amplified from pJAM1849 with the $35 \mathrm{~S}$ Ter forward and reverse primers. The wild-type sMITE (WM) was obtained by PCR with the WM forward and reverse primers. The sMITE mutants (MM.1, MM.2 and MM.3) were generated by primer-directed mutagenesis using the MM.1 forward primer, MM.2 reverse primer, and WM primers.

To replace the sMITE with other DNA fragments, a fragment from the $3^{\prime}$-UTR was amplified with the GUS.1 forward and reverse primers, and a Ty3/gypsy retrotransposon was amplified with the GUS.2 forward and reverse primers from rice genomic DNA. The Ghd2 $3^{\prime}$ UTR $\Delta \mathrm{M} / \mathrm{pDONR} 221$ fragment was amplified with the GUdM/p221 forward and reverse primers. The sMITE was substituted through blunt-end ligation between the Ghd2 $3^{\prime}$-UTR $\Delta \mathrm{M} / \mathrm{pDONR} 221$ and the replacing fragments. The three randomly chosen gene $3^{\prime}$-UTRs were amplified with the OU1-3 forward and reverse primers from rice genomic DNA.

The fragments used for transient expression were cloned into pDONR221 using the Gateway BP clonase II enzyme (Invitrogen) and were subsequently moved into p2FLGW7 with the Gateway LR clonase II enzyme (Invitrogen). The 35S Ter was cloned into p2RLGW7 for normalization.

A complete list of oligonucleotides used for plasmid preparation is in the Supplementary Table 2.

RNA ligase-mediated rapid amplification of cDNA ends. The $3^{\prime}$ UTR of Ghd 2 was amplified from the reverse-transcribed cDNA of rice tissue using RNA ligasemediated RACE (GeneRacer Kit, Invitrogen). The $3^{\prime}$ UTR was then cloned into a pGEM-T easy vector (Promega) and sequenced. The cleavage site was mapped using modified $5^{\prime}$ RNA ligase-mediated RACE with the GeneRacer Kit following the manufacturer's instructions. The ligation of an RNA oligomer to the mRNA $5^{\prime}$-end was followed by reverse transcription, amplification, cloning and sequencing. The primer sequences are listed in the Supplementary Table 2.

Quantitative RT-PCR. Total RNA was isolated from rice leaves using TRIzol Reagent (Invitrogen) in accordance with the manufacturer's instructions. A $3 \mu \mathrm{g}$ of RNA sample was subjected to deoxyribonuclease I treatment (Invitrogen) and reverse transcribed using SuperScript III Reverse Transcriptase (Invitrogen) with Oligo $(\mathrm{dT})_{18}$ or gene specific reverse primers. Quantitative RT-PCR was performed in a StepOnePlus Real-Time PCR System (Applied Biosystems) using FastStart Universal SYBR Green Master (Roche). Ubiquitin was used as a reference gene in the qRT-PCR experiments. The transcripts were quantified with the comparative $\mathrm{Ct}$ method, and differences in gene expression were presented as the normalized fold expression $(\Delta \Delta \mathrm{Ct})$. The qRT-PCR was performed three times independently. The primers used for the qRT-PCR are listed in the Supplementary Table 2.

Western blotting. Rice young leaf samples were homogenized in liquid nitrogen and lysed in $500 \mu \mathrm{l}$ of lysis buffer per $25 \mathrm{mg}$. Total proteins were separated in a $12 \%$ SDS polyacrylamide gel and were transferred to PVDF membranes (GE Healthcare). Blotting was performed with 1:2,000 anti-Ghd2 rabbit polyclonal antibody (NewEast Bioscience) and 1:1,000 anti-FLAG antibody (A2220, Sigma). Horseradish peroxidase (HRP) signals were detected after incubation with the recommended HRP-conjugated secondary antibodies by employing chemiluminescence (SuperSignal West Pico Chemiluminescent substrate, Thermo). The signal intensity was quantified with ImageJ (National Institutes of Health, http://rsb.info.nih.gov/ij/). The relative abundance was calculated from the 
quantified Ghd2 blotting signal intensity versus the quantified Rubisco signal intensity. All the western blotting was performed three times independently. Assessment of Ghd2 antibody specificity is shown in Supplementary Fig. 13. The original western blotting and gel images are provided in Supplementary Fig. 14.

Dual-luciferase reporter assay in protoplasts. The p2FLGW7-3' UTR and p2RLGW7 were co-transfected into rice protoplasts by PEG-CaCl 2 , and the protoplasts were lysed to perform a luciferase activity assay with the Dual Luciferase Reporter Assay System (Promega). In addition, RNA was isolated $14 \mathrm{~h}$ after transfection with TRIzol Reagent (Invitrogen). The RNA samples were reverse transcribed and quantified using quantitative RT-PCR. Each assay was performed three times independently in protoplasts.

Small RNA northern blotting. Total RNA was isolated from rice leaf tissues using TRIzol Reagent. Low-molecular-weight (LMW) RNA was precipitated from the supernatant after precipitation with sodium chloride and polyethylene glycol (PEG) 8,000 (ref. 67). Then, $30 \mu \mathrm{g}$ of LMW RNA was separated on a $15 \%$ denaturing PAGE gel and was transferred to a nylon membrane (GE Healthcare). Locked nucleic acid-modified probes and oligonucleotide probes (Exiqon; listed in Supplementary Table 2) were used for small RNA detection ${ }^{68}$. The signal intensity was quantified with ImageJ (National Institutes of Health, http://rsb.info.nih.gov/ij/). The relative abundance was calculated from the quantified siRNA blotting signal intensity versus quantified loading control (U6 or miR168). The original small RNA northern blotting images were provided in Supplementary Fig. 14.

Bisulfite sequencing analysis. To determine the DNA methylation levels in the MITE excision lines and the wild types, the genomic DNA was isolated from leaves at 60-day after germination using the DNeasy Plant Mini Kit (QIAGEN). Bisulfite treatment was performed with the EpiTect Bisulfite kit (Qiagen) following the manufacturer's instructions. The treated DNA was used for PCR amplification with primers at the Ghd2 locus. The primers were designed by Methyl Primer Express software (Applied Biosystems). Primers for bisulfite sequencing were listed in the Supplementary Table 2. All PCR fragments were cloned to pGEM-T easy vector (Promega) and 20 clones for each fragment were sequenced. The sequencing data were analysed on the web-based kismeth bisulfite analysis sofeware ${ }^{69}$.

Chromatin Immunoprecipitation assay. To investigate the histone modification at the Ghd2 locus in the MITE excision lines and the segregated wild types, chromatin immunoprecipitation (ChIP) assays were carried out in the mature leaves as previously described ${ }^{70}$. The antibodies for anti-H3K9 dimethylation (Abcam A1220) and mouse IgG2 (abcam 18413) were employed in the ChIP followed by qPCR. For validation of the ChIP results of H3K9me2, a rice Ty1-copia retrotransposon (Os02g30880) and the actin gene (Os11g06390) were used as positive and negative controls, respectively. The primers for ChIP-qPCR were listed in the Supplementary Table 2.

Statistical analysis. The two-tailed Student's $t$-test was used for statistical analysis whenever two groups were compared. Statistical significance was determined at $P<0.05\left(^{*}\right)$ or $P<0.01\left({ }^{*}\right)$

Data availability. The sequences of p2FLGW7 and p2RLGW7 have been submitted to Genbank under accession code (KY434118 and KY434119). The authors declare that all data supporting the findings of this study are available within the manuscript and its supplementary files or are available from the corresponding author on request.

\section{References}

1. Huang, C. R., Burns, K. H. \& Boeke, J. D. Active transposition in genomes. Annu. Rev. Genet. 46, 651-675 (2012).

2. Bennetzen, J. L. \& Wang, H. The contributions of transposable elements to the structure, function, and evolution of plant genomes. Annu. Rev. Plant Biol. 65, 505-530 (2014).

3. Wicker, T. et al. A unified classification system for eukaryotic transposable elements. Nat. Rev. Genet. 8, 973-982 (2007).

4. Bureau, T. E., Ronald, P. C. \& Wessler, S. R. A computer-based systematic survey reveals the predominance of small inverted-repeat elements in wild-type rice genes. Proc. Natl Acad. Sci. USA 93, 8524-8529 (1996).

5. Santiago, N., Herraiz, C., Goni, J. R., Messeguer, X. \& Casacuberta, J. M. Genome-wide analysis of the Emigrant family of MITEs of Arabidopsis thaliana. Mol. Biol. Evol. 19, 2285-2293 (2002).

6. Ostertag, E. M. \& Kazazian, H. H. Genetics: LINEs in mind. Nature 435, 890-891 (2005).

7. Kramerov, D. A. \& Vassetzky, N. S. Short retroposons in eukaryotic genomes. Int. Rev. Cytol. 247, 165-221 (2005).
8. Dewannieux, M., Esnault, C. \& Heidmann, T. LINE-mediated retrotransposition of marked Alu sequences. Nat. Genet. 35, 41-48 (2003).

9. Elbarbary, R. A., Lucas, B. A. \& Maquat, L. E. Retrotransposons as regulators of gene expression. Science 351, aac7247 (2016).

10. Ichiyanagi, K. Epigenetic regulation of transcription and possible functions of mammalian short interspersed elements, SINEs. Genes Genet. Syst. 88, 19-29 (2013).

11. Estecio, M. R. et al. SINE retrotransposons cause epigenetic reprogramming of adjacent gene promoters. Mol. Cancer Res. 10, 1332-1342 (2012).

12. Sasaki, T. et al. Possible involvement of SINEs in mammalian-specific brain formation. Proc. Natl Acad. Sci. USA 105, 4220-4225 (2008).

13. Faulkner, G. J. et al. The regulated retrotransposon transcriptome of mammalian cells. Nat. Genet. 41, 563-571 (2009).

14. Sorek, R., Ast, G. \& Graur, D. Alu-containing exons are alternatively spliced. Genome Res. 12, 1060-1067 (2002).

15. Lev-Maor, G., Sorek, R., Shomron, N. \& Ast, G. The birth of an alternatively spliced exon: $3^{\prime}$ splice-site selection in Alu exons. Science 300, 1288-1291 (2003).

16. Roy-Engel, A. M. et al. Human retroelements may introduce intragenic polyadenylation signals. Cytogenet. Genome Res. 110, 365-371 (2005).

17. Lee, J. Y., Ji, Z. \& Tian, B. Phylogenetic analysis of mRNA polyadenylation sites reveals a role of transposable elements in evolution of the $3^{\prime}$-end of genes. Nucleic Acids Res. 36, 5581-5590 (2008).

18. Chen, L. L., DeCerbo, J. N. \& Carmichael, G. G. Alu element-mediated gene silencing. EMBO J. 27, 1694-1705 (2008).

19. An, H. J., Lee, D., Lee, K. H. \& Bhak, J. The association of Alu repeats with the generation of potential AU-rich elements (ARE) at $3^{\prime}$ untranslated regions. BMC Genomics 5, 97 (2004).

20. Elbarbary, R. A., Li, W., Tian, B. \& Maquat, L. E. STAU1 binding $3^{\prime}$ UTR IRAlus complements nuclear retention to protect cells from PKR-mediated translational shutdown. Genes Dev. 27, 1495-1510 (2013).

21. Elbarbary, R. A. \& Maquat, L. E. Dodging two bullets with one dsRNA-binding protein. Cell Cycle 13, 345-346 (2014).

22. Capshew, C. R., Dusenbury, K. L. \& Hundley, H. A. Inverted Alu dsRNA structures do not affect localization but can alter translation efficiency of human mRNAs independent of RNA editing. Nucleic Acids Res. 40, 8637-8645 (2012).

23. Fitzpatrick, T. \& Huang, S. $3^{\prime}$-UTR-located inverted Alu repeats facilitate mRNA translational repression and stress granule accumulation. Nucleus $\mathbf{3}$, 359-369 (2012).

24. Kim, Y. et al. PKR is activated by cellular dsRNAs during mitosis and acts as a mitotic regulator. Genes Dev. 28, 1310-1322 (2014).

25. Liu, W. M., Chu, W. M., Choudary, P. V. \& Schmid, C. W. Cell stress and translational inhibitors transiently increase the abundance of mammalian SINE transcripts. Nucleic Acids Res. 23, 1758-1765 (1995).

26. Carrieri, C. et al. Long non-coding antisense RNA controls Uchl1 translation through an embedded SINEB2 repeat. Nature 491, 454-457 (2012).

27. Jiang, N., Feschotte, C., Zhang, X. \& Wessler, S. R. Using rice to understand the origin and amplification of miniature inverted repeat transposable elements (MITEs). Curr. Opin. Plant Biol. 7, 115-119 (2004).

28. Naito, K. et al. Unexpected consequences of a sudden and massive transposon amplification on rice gene expression. Nature 461, 1130-1134 (2009).

29. Wei, L. et al. Dicer-like 3 produces transposable element-associated 24-nt siRNAs that control agricultural traits in rice. Proc. Natl Acad. Sci. USA 111, 3877-3882 (2014).

30. Mao, H. et al. A transposable element in a NAC gene is associated with drought tolerance in maize seedlings. Nat. Commun. 6, 8326 (2015).

31. Wu, L. et al. DNA methylation mediated by a microRNA pathway. Mol. Cell 38, 465-475 (2010).

32. Nosaka, M. et al. Role of transposon-derived small RNAs in the interplay between genomes and parasitic DNA in rice. PLoS Genet. 8, e1002953 (2012).

33. Strayer, C. et al. Cloning of the Arabidopsis clock gene TOC1, an autoregulatory response regulator homolog. Science 289, 768-771 (2000).

34. Kaczorowski, K. A. \& Quail, P. H. Arabidopsis PSEUDO-RESPONSE REGULATOR7 is a signaling intermediate in phytochrome-regulated seedling deetiolation and phasing of the circadian clock. Plant Cell 15, 2654-2665 (2003).

35. Yan, L. et al. The wheat VRN2 gene is a flowering repressor down-regulated by vernalization. Science 303, 1640-1644 (2004).

36. Xue, W. et al. Natural variation in Ghd7 is an important regulator of heading date and yield potential in rice. Nat. Genet. 40, 761-767 (2008).

37. Hung, H. Y. et al. ZmCCT and the genetic basis of day-length adaptation underlying the postdomestication spread of maize. Proc. Natl Acad. Sci. USA 109, E1913-E1921 (2012).

38. Yang, Q. et al. CACTA-like transposable element in ZmCCT attenuated photoperiod sensitivity and accelerated the postdomestication spread of maize. Proc. Natl Acad. Sci. USA 110, 16969-16974 (2013). 
39. Griffiths, S., Dunford, R. P., Coupland, G. \& Laurie, D. A. The evolution of CONSTANS-like gene families in barley, rice, and Arabidopsis. Plant Physiol. 131, 1855-1867 (2003).

40. McCouch, S. Gene nomenclature system for rice. Rice 1, 72-84 (2008).

41. Lu, C. et al. Miniature inverted-repeat transposable elements (MITEs) have been accumulated through amplification bursts and play important roles in gene expression and species diversity in Oryza sativa. Mol. Biol. Evol. 29, 1005-1017 (2012).

42. Doi, K. et al. Ehd1, a B-type response regulator in rice, confers short-day promotion of flowering and controls FT-like gene expression independently of Hd1. Genes Dev. 18, 926-936 (2004).

43. Yano, M. et al. Hd1, a major photoperiod sensitivity quantitative trait locus in rice, is closely related to the Arabidopsis flowering time gene CONSTANS. Plant Cell 12, 2473-2484 (2000).

44. Liu, J., He, Y., Amasino, R. \& Chen, X. siRNAs targeting an intronic transposon in the regulation of natural flowering behavior in Arabidopsis. Genes Dev. 18, 2873-2878 (2004).

45. Swiezewski, S. et al. Small RNA-mediated chromatin silencing directed to the $3^{\prime}$ region of the Arabidopsis gene encoding the developmental regulator, FLC. Proc. Natl Acad. Sci. USA 104, 3633-3638 (2007).

46. Ma, X. et al. Small interfering RNA-mediated translation repression alters ribosome sensitivity to inhibition by cycloheximide in Chlamydomonas reinhardtii. Plant Cell 25, 985-998 (2013).

47. Johnson, C., Bowman, L., Adai, A. T., Vance, V. \& Sundaresan, V. CSRDB: a small RNA integrated database and browser resource for cereals. Nucleic Acids Res. 35, D829-D833 (2007).

48. Nagano, H., Fukudome, A., Hiraguri, A., Moriyama, H. \& Fukuhara, T. Distinct substrate specificities of Arabidopsis DCL3 and DCL4. Nucleic Acids Res. 42, 1845-1856 (2014).

49. Xie, Z. et al. Genetic and functional diversification of small RNA pathways in plants. PLoS Biol. 2, E104 (2004).

50. Zilberman, D. et al. Role of Arabidopsis ARGONAUTE4 in RNA-directed DNA methylation triggered by inverted repeats. Curr. Biol. 14, 1214-1220 (2004).

51. Zilberman, D., Cao, X. \& Jacobsen, S. E. ARGONAUTE4 control of locusspecific siRNA accumulation and DNA and histone methylation. Science 299, 716-719 (2003).

52. Chan, S. W. et al. RNA silencing genes control de novo DNA methylation. Science 303, 1336 (2004).

53. Chen, J., Hu, Q., Zhang, Y., Lu, C. \& Kuang, H. P-MITE: a database for plant miniature inverted-repeat transposable elements. Nucleic Acids Res. 42, D1176-D1181 (2014).

54. Fattash, I. et al. Miniature inverted-repeat transposable elements: discovery, distribution, and activity. Genome 56, 475-486 (2013).

55. Hiei, Y. \& Komari, T. Agrobacterium-mediated transformation of rice using immature embryos or calli induced from mature seed. Nat. Protoc. 3, 824-834 (2008).

56. Song, X. et al. Roles of DCL4 and DCL3b in rice phased small RNA biogenesis. Plant J. 69, 462-474 (2012).

57. Liu, B. et al. Loss of function of OsDCL1 affects microRNA accumulation and causes developmental defects in rice. Plant Physiol. 139, 296-305 (2005).

58. Zhang, J. et al. Combinations of the Ghd7, Ghd8 and Hd1 genes largely define the ecogeographical adaptation and yield potential of cultivated rice. New Phytol. 208, 1056-1066 (2015).

59. Xie, K. \& Yang, Y. RNA-guided genome editing in plants using a CRISPR-Cas system. Mol. Plant 6, 1975-1983 (2013).

60. Xiao, B., Huang, Y., Tang, N. \& Xiong, L. Over-expression of a LEA gene in rice improves drought resistance under the field conditions. Theor. Appl. Genet. 115, 35-46 (2007)

61. Miao, J. et al. Targeted mutagenesis in rice using CRISPR-Cas system. Cell Res. 23, 1233-1236 (2013).

62. Xie, K., Zhang, J. \& Yang, Y. Genome-wide prediction of highly specific guide RNA spacers for CRISPR-Cas9-mediated genome editing in model plants and major crops. Mol. Plant 7, 923-926 (2014).

63. Xie, K., Minkenberg, B. \& Yang, Y. Boosting CRISPR/Cas9 multiplex editing capability with the endogenous tRNA-processing system. Proc. Natl Acad. Sci. USA 112, 3570-3575 (2015)
64. Schwab, R., Ossowski, S., Riester, M., Warthmann, N. \& Weigel, D. Highly specific gene silencing by artificial microRNAs in Arabidopsis. Plant Cell 18, 1121-1133 (2006).

65. Warthmann, N., Chen, H., Ossowski, S., Weigel, D. \& Herve, P. Highly specific gene silencing by artificial miRNAs in rice. PLOS ONE 3, e1829 (2008).

66. Cao, Y. et al. The expression pattern of a rice disease resistance gene xa3/xa26 is differentially regulated by the genetic backgrounds and developmental stages that influence its function. Genetics 177, 523-533 (2007).

67. Dalmay, T., Hamilton, A., Mueller, E. \& Baulcombe, D. C. Potato virus X amplicons in arabidopsis mediate genetic and epigenetic gene silencing. Plant Cell 12, 369-379 (2000).

68. Varallyay, E., Burgyan, J. \& Havelda, Z. MicroRNA detection by northern blotting using locked nucleic acid probes. Nat. Protoc. 3, 190-196 (2008).

69. Gruntman, E. et al. Kismeth: analyzer of plant methylation states through bisulfite sequencing. BMC Bioinformatics 9, 371 (2008).

70. Lawrence, R. J. et al. A concerted DNA methylation/histone methylation switch regulates rRNA gene dosage control and nucleolar dominance. Mol. Cell 13, 599-609 (2004).

\section{Acknowledgements}

We thank Dr Yijun Qi and Dr Xiaofeng Cao for providing the siRNA biogenesis-related constructs and rice materials and Dr Qifa Zhang and Dr Yongzhong Xing for providing the wild rice materials and rice cultivars DNA. We thank Dr Yijun Qi and Dr Jing Ning for their helpful discussions, Dr Xiaozhuo Wang for processing the images, Dr Renjie Tang for help with revising the manuscript. This work was supported by grants from the National Key Project of Research and Development on Rice Functional Genomics (2016YFD0100900) and the National Program of China for Transgenic Research (2014ZX08009-003-002, 2014ZX08001-003)

\section{Author contributions}

J.S. designed and performed the experiments, analysed the results, and wrote the manuscript; J.L. prepared the Ghd 2 over-expression and CRISPR lines, and determined the phenotypes. K.X. designed the gRNAs for the sMITE excision, discussed the results, and revised the paper. F. Xing performed genome-wide identification of $3^{\prime}$-UTR-localized MITEs. F. Xiong cultivated the transgenic lines. J.X. and X.L. provided the reagents and management. L.X. designed the experiments, analysed the results and wrote the manuscript.

\section{Additional information}

Supplementary Information accompanies this paper at http://www.nature.com/ naturecommunications

Competing financial interests: The authors declare no competing financial interests.

Reprints and permission information is available online at http://npg.nature.com/ reprintsandpermissions/

How to cite this article: Shen, J. et al. Translational repression by a miniature invertedrepeat transposable element in the $3^{\prime}$ untranslated region. Nat. Commun. 8, 14651 doi: 10.1038/ncomms14651 (2017).

Publisher's note: Springer Nature remains neutral with regard to jurisdictional claims in published maps and institutional affiliations.

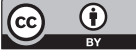

This work is licensed under a Creative Commons Attribution 4.0 International License. The images or other third party material in this article are included in the article's Creative Commons license, unless indicated otherwise in the credit line; if the material is not included under the Creative Commons license, users will need to obtain permission from the license holder to reproduce the material. To view a copy of this license, visit http://creativecommons.org/licenses/by/4.0/

(C) The Author(s) 2017 